\title{
SOME DERIVATIONS ON LEVY AND JUMP PROCESSES
}

\author{
Md. Sharif Ullah Mozumder \\ Department of Mathematics, University of Dhaka, Dhaka, Bangladesh \\ sharif_math2000@yahoo.com
}

Received 22.08.06 Accepted 30.08.08

\begin{abstract}
Modeling with jump processes has become an integral part of real life mathematics. Besides actuarial applications jump processes have frequently been applied in credit risk modeling, derivative pricing, interest rate modeling, signal processing and so on. Behind every jump process there always exists a stochastic process. Once this stochastic process satisfies some conditions (to be mentioned) it is known as Lévy process. This particular family of stochastic processes has rich structures of its own which facilitate the study of jump processes associated with the processes belonging to this family. In this article some results, involving both Lévy and jump processes, are proved in details. These details derivations, explicitly showing the intuitive meaning of Lévy properties, are not available in the existing literature.
\end{abstract}

\section{Introduction}

It is natural to expect that in any jump process the stochastic process starting at zero, with which the jump process is associated, will have independent random variables describing the increments on non-overlapping intervals. This is known as the independent increments property. The distribution of these random increments depends only on the length of the corresponding intervals but not on their starting point, i.e. the distribution is stationary in the sense that it remains the same for intervals of same length no matter where the intervals occur. This is known as the stationary increments property. Also the process is expected to be continuous, a property known as stochastic continuity. A process satisfying all these three properties is known as Lévy process. Many processes in application, namely Brownian motion, the compound Poisson process (including the Poisson process as a special case), stable process do satisfy these three conditions as will be shown in case of the compound Poisson and Poisson. Conversely it's been discussed what more condition(s) a process having these three properties should satisfy in order to be Poisson and compound Poisson. But the jump processes associated with these processes are not assured to satisfy these conditions. In fact it's shown that these jump processes are not straightforward to analyze. Lévy properties help us when dealing with the jump processes associated with the Lévy processes.

\section{Preliminaries and definitions}

In this section we will get necessary definitions (for a complete description of Lévy theory see [3] and [4]). Consider a measurable space $(\Omega, F, \mu)$ where the measure $\mu$ is a set function defined as 


$$
\mu: \mathcal{F} \rightarrow R^{+}
$$

In case $\mu(\Omega)=1, \mu$ is denoted as ' $P$ ' and is known as probability measure on $\Omega$.

Definition 2.1 A function $f:[0, T] \rightarrow R \quad$ is said to be cadlag if it is right continuous with left limits (rcll) i.e. for each $t \in[0, T]$ the limits

$$
f\left(t^{-}\right)=\lim _{\substack{s \rightarrow t \\ s<t}} f(s) \text { and } f\left(t^{+}\right)=\lim _{\substack{s \rightarrow t \\ s>t}} f(s)
$$

exist and

$$
f(t)=f\left(t^{+}\right) .
$$

So clearly any continuous function is cadlag but cadlag functions can have jumpdiscontinuities (only).If $t$ is a point of discontinuity of $f$, then the jump of $f$ at $t$ is defined and denoted as

$$
\Delta f(t)=f(t)-f\left(t^{-}\right)
$$

Cadlag functions cannot jump around too wildly as indicated by the following proposition:

Proposition 2.1 A cadlag function can have at most a countable number of discontinuities. i.e. the set

$$
\left\{t \in[0, T]: f(t) \neq f\left(t^{-}\right)\right\}=\{t \in[0, T]: \Delta f(t) \neq 0\}
$$

is either finite or atmost countable.

The above proposition is, in fact, one of the corner stones of stochastic calculus. For a nice detailed proof and other facts about cadlag functions the readers are referred to [2].

It can be shown that for any $\varepsilon>0$, the number of jumps on $[0, T]$ which are larger than $\varepsilon>0$ should be finite. See [5].

Hence a cadlag function on $[0, T]$ has a finite number of large jumps

(larger than $\varepsilon$ )and possibly an infinite but countable number of small jumps.

Definition 2.2 Let $X=\left(X_{t}, t \geq 0\right)$ is a cadlag stochastic process. Its associated jump process, denoted as $\Delta X=\left(\Delta X_{t}, t \geq 0\right)$, is defined by

$$
\Delta X_{t}=X_{t}-X_{t}-
$$

for each $t \geq 0$, where $X_{t}$ - is the left limit at the point $t$.

Definition 2.3 Let $X$ be a random variable defined on $(\Omega, F, P)$ taking values in $\mathfrak{R}$. Its characteristic function $\Phi: \mathfrak{R} \rightarrow$ is defined, $t \in \mathfrak{R}$, by

$$
\Phi_{X}(t)=E\left(e^{i t X}\right)=\int_{R} e^{i t x} F_{X}(d x)=\int_{R} e^{i t x} d F=\int_{-\infty}^{\infty} e^{i t x} f(x) d x,
$$


where $F_{X}(x)=\boldsymbol{P} o X^{-1}(-\infty, x]$.

Remark 2.1 From the definition it follows that if $X$ and $Y$ are independent random variables then

$$
\Phi_{X+Y}(t)=\Phi_{X}(t) \Phi_{Y}(t)
$$

Let $N$ be a Poisson random variable with parameter $\alpha$ i.e.

$$
\mathbf{P}[N=n]=\frac{e^{-\alpha} \cdot \alpha^{n}}{n !}
$$

Then the characteristic function of $N$ is:

$$
\Phi_{N}(t)=\sum_{n=0}^{\infty} e^{i t n} \frac{e^{-\alpha} \cdot \alpha^{n}}{n !}=e^{-\alpha} \sum_{n=0}^{\infty} \frac{\left[\alpha \cdot e^{i t}\right]^{n}}{n !}=e^{-\alpha} \cdot e^{\alpha e^{i t}}=e^{\alpha\left[e^{i t}-1\right]} .
$$

Definition 2.4 Let $\left\{\tau_{i}\right\}_{i \geq 1}$ be a sequence of independent exponential random variables with parameter $\alpha$ and $T_{n}=\sum_{i=1}^{n} t_{i}$. The processes $N_{t}$ defined by

$$
N_{t}=\sum_{n \geq 1} 1_{\left[t \geq T_{n}\right]}
$$

is called a Poisson process with intensity $\alpha$.

The Poisson process, therefore, counts the number of random times $T_{n}$ which occur between 0 and t, where $\left\{T_{n}-T_{n-1}\right\}_{n \geq 1}$ is an i.i.d. sequence of exponential variables. The following proposition explains the use of the term "Poisson".

Proposition 2.2 If $\left(t_{i}\right)_{i^{3} 1}$ are independent exponential random variables with parameter $\alpha$ then for any $t>0$ the random variable

$$
N_{t}=\inf \left\{n \geq 1, \sum_{i=1}^{n} \tau_{i}>t\right\}
$$

follows a Poisson distribution with parameter $\alpha t$ :

$$
P\left(N_{t}=n\right)=e^{-a t} \frac{(a t)^{n}}{n !} \quad \forall n \in \mathrm{N} .
$$

A nice intuitive proof can be found e.g.in [1].

From (3) and (6) it follows that the characteristic function of a Poisson process is given by

$$
E\left[e^{i u N_{t}}\right]=\exp \left\{a t\left(e^{i u}-1\right)\right\}, \quad \forall u \in \Re
$$


Remark 2.2 For any $\omega$ the sample path $t \rightarrow N_{t}(\omega)$ is piecewise constant and from (4) it is clear that the path increases by jumps of size 1

Definition 2.5 A compound Poisson process with intensity $\alpha>0$ is a stochastic process $X_{t}$ defined as

$$
X_{t}=\sum_{n=1}^{N_{t}} Z_{n}=Z_{1}+\cdots+Z_{N_{t}}
$$

where jump sizes $Z_{n}$ are i.i.d. with distribution $F_{Z}$ and $N_{t}$ is a Poisson process with intensity $\alpha$ independent of $\left\{Z_{n}\right\}_{n \geq 1}$

Details on compound Poisson processes can be found in [1]. A simple derivation gives the characteristic function of a compound Poisson process:

$$
E\left[e^{i u X} t\right]=\exp \left\{t \alpha \int_{-\infty}^{\infty}\left(e^{i u x}-1\right) F_{Z}(d x)\right\} \quad \forall t \in R
$$

where $\alpha$ denotes the jump intensity and $F$ the jump size distribution. See e.g. [1].

Definition 2.6 $A$ cadlag stochastic process $\left\{X_{t}, t \geq 0\right\}$, on $(\Omega, F, P)$ with values in $R$ is called a Lévy process if it satisfies the following properties:

[L1] each $X_{0}=0$ a.s.(i.e.with probability 1)

[L2] $X_{t}$ has independent and stationary increments i.e.,

(i) for every increasing sequence of times $t_{0}<t_{1}<t_{2}<\cdots<t_{n}$ the random variables $X_{t_{0}}, X_{t_{1}}-X_{t_{0}}, \cdots, X_{t_{n}}-X_{t_{n-1}} \quad$ are independent,

(ii) $X_{t+h}-X_{t=}^{d} X_{(t+h-t)}=X_{h}$ i.e.the distribution of $X_{t+h}-X_{t}$ does not depend on $t$,

[L3] $X_{t}$ is stochastically continuous i.e.

$$
\lim _{h \rightarrow 0} \boldsymbol{P}\left(\left|X_{t+h}-X_{t}\right|>\varepsilon\right)=0, \quad \forall \varepsilon>0 .
$$

Property [L3] doesn't imply the continuity of the sample paths. The intuitive meaning of [L3] is that for a given time $t$ (deterministic) the probability of having a jump at $t$ is zero, i.e. discontinuities (jumps) do not occur at deterministic times and so occur at random times. It serves to exclude processes with jumps at fixed times.

Proposition 2.3 If $X=\left(X_{t}, t \geq 0\right)$ is a Lévy process, then for fixed $t>0, \Delta X_{t}=0$ a.s. 
Proof: Consider a sequence $\left(t_{n}, n \in N\right)$ in $R^{+}$with $t_{n} \square t$ as $n \rightarrow \infty$. Since $X$ has cadlag paths. However by [L3] the sequence $\left(X_{t_{n}}, n \in N\right)$ converges in probability to $X_{t}$ and so has a subsequence which converges almost surely to $X_{t}$. Hence the result follows from the definition of jumps and the uniqueness of the limit.

Remark 2.3 The above proposition shows that $\Delta_{X}$ is not a straightforward process to analyze.

Definition 2.7 A standard Brownian motion in $\mathfrak{R}$ is a Lévy process $B=\left(B_{t}, t \geq 0\right)$ for which:

$$
\text { [B1] } B_{t} \sim N(0, t) \quad \text { for each } t \geq 0,
$$

[B2] B has continuous sample paths .

In fact Brownian motion is the only Lévy process having continuous sample paths, see [3]. From [B1] and (1) it follows immediately that Brownian motion has a characteristic function given by:

$$
\Phi_{B_{t}}(u)=\exp \left\{-\frac{1}{2} t u^{2}\right\}, \quad \text { for all } u \in R \text { and } t \geq 0 \text {. }
$$

By an adapted process we mean that for each increasing sequence $\left\{t_{n}\right\}_{n \geq 1}$ there is an increasing family $F_{t_{n}}$ of sub $\sigma$-algebras of $F$ with the property that for each $t \geq 0$ we can find $F_{t}$ so that $X_{t}$ is $F_{t}$ - measurable. Such a family $\left\{F_{t_{n}}\right\}$ is known as a filtration.

Definition 2.8 A stopping time is a random variable $T: \Omega \rightarrow[0, \infty]$ for which the event $(T \leq t) \in F_{t}$ for each $t \geq 0$.

Any ordinary deterministic time is clearly a stopping time.An intuitive example, which has many important applications, is the first hitting time $T_{A}$ of a process to a Borel set $A$ in $\mathfrak{R}$.This is defined ,for an $F_{t}$ adapted cadlag process, as

$$
T_{A}=\inf \left\{t \geq 0 \mid X_{t} \in A\right\}
$$

with the convention that $\inf \{\varnothing\}=\infty$.

Definition 2.9 (Convolution) The convolution of the distribution functions $F_{1}$ and $F_{2}$ on $R$ is defined as

$$
\left(F_{1} * F_{2}\right)(x)=\int_{-\infty}^{\infty} F_{1}(x-y) d F_{2}(y), \quad \forall x \in R
$$


Remark 2.4 The above convolution describes the distribution of $X_{1}+X_{2}$ :

$$
F_{X_{1}+X_{2}}(u)=\int_{-\infty}^{\infty} F_{1}(u-y) d F_{2}(y)
$$

\section{Results of interest from the theory Lévy processes}

In this section detailed proofs of some results from Lévy processes, which are related with the jump processes in application, are presented.

Theorem 3.1 The compound Poisson process $X_{t}=\sum_{i=i}^{N_{t}} Z_{i}$ is a Lévy process, where $N_{t}$ is a Poisson process with intensity $\alpha$ and $Z_{i}$ 's are i.i.d. random variables independent of $N$.

Proof: $N_{0}=0$ a.s. (by the definition of a Poisson process) implies that $X_{0}=0$ a.s. For $0=t_{0}<t_{1}<t_{2} \cdots<t_{n}$

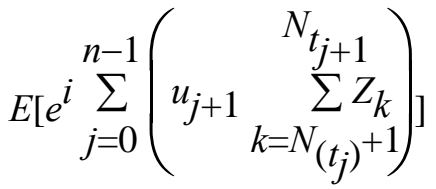

$$
\begin{aligned}
& =\sum_{i_{1}=0}^{\infty} \cdots \sum_{i_{n}=0}^{\infty} E\left[e^{i} \sum_{j=0}^{n-1}\left(u_{j+1} \begin{array}{r}
\sum_{t_{j+1}} \\
\sum_{k=N_{\left(t_{j}\right)}+1} Z_{k}
\end{array}\right) \mid N_{t_{1}}=i_{1}, N_{t_{2}}=i_{1}+i_{2}, \cdots,\right. \\
& \left.N_{t_{n}}=i_{1}+\cdots+i_{n}\right] P\left(N_{t_{1}}=i_{1}, N_{t_{2}}=i_{1}+i_{2}, \cdots, N_{t_{n}}=i_{1}+\cdots+i_{n}\right) \\
& =\sum_{i_{1}=0}^{\infty} \cdots \sum_{i_{n}=0}^{\infty} \Phi_{Z}^{i_{1}}\left(u_{1}\right) \cdots \Phi_{Z}^{i_{n}}\left(u_{n}\right) P\left(N_{t_{1}}-N_{t_{0}}=i_{1}, \cdots, N_{t_{n}}-N_{t_{n-1}}=i_{n}\right) \\
& =\sum_{i_{1}=0}^{\infty} \cdots \sum_{i_{n}=0}^{\infty} \Phi_{Z}^{i_{1}}\left(u_{1}\right) \cdots \Phi_{Z}^{i_{n}}\left(u_{n}\right) P\left(N_{t_{1}}-N_{t_{0}}=i_{1}\right) \cdots P\left(N_{t_{n}}-N_{t_{n-1}}=i_{n}\right)
\end{aligned}
$$

Now computing the same quantity in another way we get:

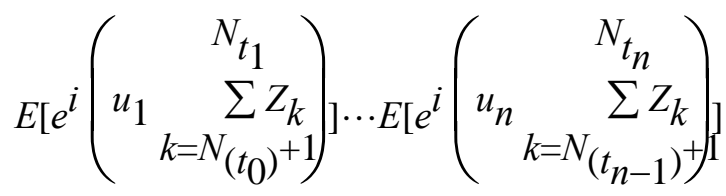




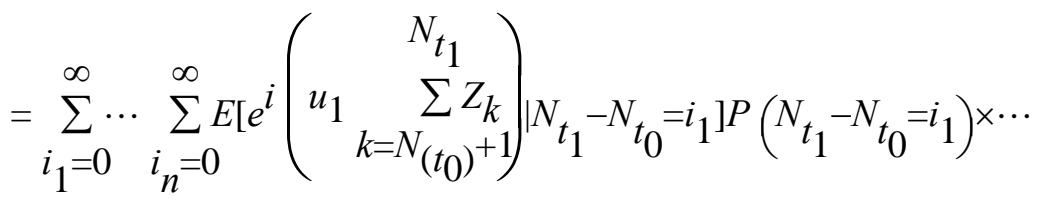

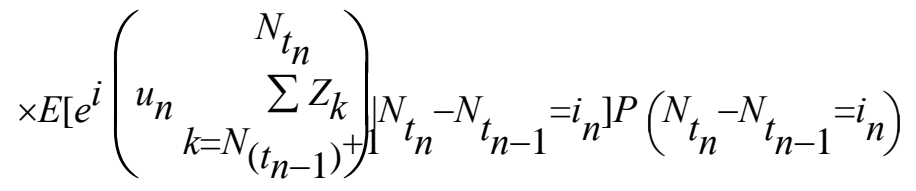

$$
\begin{aligned}
& =\sum_{i_{1}=0}^{\infty} \cdots \sum_{i_{n}=0}^{\infty} \Phi_{Z}^{i_{1}}\left(u_{1}\right) \cdots \Phi_{Z}^{i_{n}}\left(u_{n}\right) P\left(N_{t_{1}}-N_{t_{0}}=i_{1}\right) \cdots P\left(N_{t_{n}}-N_{t_{n-1}}=i_{n}\right)
\end{aligned}
$$

So from (10) and (11) we get that $X_{t_{1}}-X_{t_{0}}, X_{t_{2}}-X_{t_{1}} \cdots X_{t_{n}}-X_{t_{n-1}}$ are independent.

Now to show stationarity we proceed as follows: Let $0 \leq s<t$. Then

$$
\begin{aligned}
& E\left[e^{i\left(u, X_{t}-X_{S}\right)}\right] \\
& =E\left[e^{i\left(u \sum_{k=N_{S}+1}^{N_{t}} Z_{k}\right)}\right]
\end{aligned}
$$

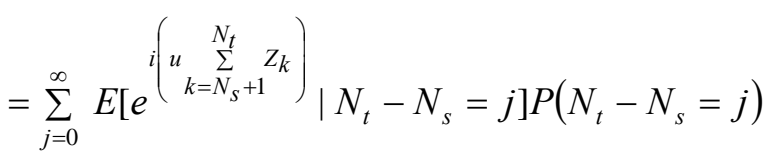

$$
\begin{aligned}
& =\sum_{j=0}^{¥} F_{Z}^{j}(u) P\left(N_{t}-N_{s}=j\right) \\
& =\sum_{j=0}^{\infty} F_{Z}^{j}(u) P\left(N_{t-s}=j\right)[\text { by the definition of the Poisson process }] \\
& =E\left[e^{i\left(u \sum_{k=1}^{N_{t-s}} Z_{k}\right)}\right]=\mathrm{E}\left\lfloor e^{i\left(u X_{t-8}\right.}\right\rfloor
\end{aligned}
$$

which shows that $X_{t}-X_{s}{ }^{D} X_{t-s}$.

Finally to show the stochastic continuity: consider for any $a>0$ and $t>0$

$$
\begin{aligned}
& P\left(\left|X_{t}\right|>a\right)=\sum_{i=1}^{\infty} P\left(\left|Z_{1}+Z_{2}+\cdots+Z_{i}\right|>a \mid N_{t}=i\right) P\left(N_{t}=i\right) \\
& =\sum_{i=1}^{\infty} P\left(\left|Z_{1}+Z_{2}+\right|>a\right) P\left(N_{t}=i\right)
\end{aligned}
$$




$$
\leq 1-e^{-\alpha t} \longrightarrow 0 \quad \text { as } t \longrightarrow 0
$$

where the last equality holds since $Z_{n}$ and $N$ are independent. Hence compound Poisson process is a Lévy process.

The opposite implication is conditionally true, leading to the following theorem.

Theorem 3.2 $X=\left(X_{t}, t \geq 0\right)$ is a compound Poisson process if and only if it is a Lévy process and its sample paths are piecewise constant functions.

Thus to conclude whether a general Lévy process is a compound Poisson process or not it is sufficient to observe whether its sample paths are piecewise constant or not. In fact the compound Poisson process is the only(considering the Poisson process as the simplest compound Poisson process) Lévy process with piecewise constant sample paths.

One implication of the above theorem has just been proved. The other implication can be found in [1].

Now since the Poisson process can be treated as the simplest compound Poisson process the following result is just a corollary of the above theorem.

Corollary 3.1 The poisson process is a Lévy process.

It is known that Brownian motion is, by definition, a Lévy process and its just shown that the compound Poisson process is also a Lévy process. The sum of an independent Brownian motion and a compound Poisson process is a jump-diffusion process. The following theorem shows that this jump diffusion process is also a Lévy process and hence can be separately termed as Lévy jump-diffusion process (there are jump-diffusion processes which are not Lévy processes).

Theorem 3.3 The sum of two independent Lévy processes is also a Lévy process.

The following lemma is useful in proving the above theorem.

Lemma 3.1 The random variables $X_{1}, \cdots, X_{n}$ are independent if and only if

$$
E\left(e^{i} \sum_{j=1}^{n} u_{j} X_{j}\right)=\Phi_{X_{1}}\left(u_{1}\right) \cdots \Phi_{X_{n}}\left(u_{n}\right)
$$

for all $u_{1}, \cdots, u_{n} \in R$

Applying this lemma the proof of Theorem 3.3 follows.

Proof: Let $X_{t}$ and $Y_{t}$ be two independent Lévy processes and consider

$$
Z_{t}=X_{t}+Y_{t}
$$

By [L1] $Z_{0}=X_{0}+Y_{0}=0$ a.s.. To prove the independence of the increments let for any $n \in N$, $0=t_{0}<t_{1}<\cdots<t_{n}<\infty$. Then 


$$
\begin{aligned}
& E\left[e^{i}\left(\sum_{k=0}^{n-1} u_{k+1}\left(Z_{t_{k+1}}-Z_{t_{k}}\right)\right)\right] \\
& =E\left[e^{i}\left(\sum_{k=0}^{n-1} u_{k+1}\left(X_{t_{k+1}}-X_{t_{k}}+Y_{t_{k+1}}-Y_{t_{k}}\right)\right]\right. \\
& =E\left[e^{i}\left(\sum_{k=0}^{n-1} u_{k+1}\left(X_{t_{k+1}}-X_{t_{k}}\right)\right] E\left[e^{i}\left(\sum_{k=0}^{n} u_{k+1}\left(Y_{t_{k+1}}-Y_{t_{k}}\right)\right)\right]\right. \\
& =\prod_{k=0}^{n-1} F_{X_{t_{k+1}}-X_{t_{k}}}\left(u_{k+1}\right) \prod_{k=0}^{n-1} F_{Y_{t_{k+1}}-Y_{t_{k}}}\left(u_{k+1}\right) \quad \text { [by[L2](i)and(12)] } \\
& =\prod_{k=0}^{n-1} F_{X_{t_{k+1}}-X_{t_{k}}+Y_{t_{k+1}}-Y_{t_{k}}}\left(u_{k+1}\right) \quad \text { forallu }{ }_{1}, \ldots \ldots ., \mathrm{u}_{\mathrm{n}} \in \mathfrak{R}[\mathrm{by}(2)] \\
& =\prod_{k=0}^{n-1} \Phi_{Z_{t_{k+1}}}-Z_{t_{k}}\left(u_{k+1}\right)
\end{aligned}
$$

and considering (12), again, this implies that $Z_{t}$ has independent increments. Now to show that $Z_{t}$ has stationary increments we proceed as follows:

Let $\Delta X_{j}=X_{t_{j+1}}-X_{t_{j}}$ and $\Delta Y_{j}=Y_{t_{j+1}}-Y_{t_{j}}$. Since $X_{t}$ and $Y_{t}$ have stationary increments it follows that:

$$
F_{\Delta X_{j}}=F_{X_{\left(t_{j+1}-t_{j}\right)}} \text { and } F_{\Delta Y_{j}}=F_{Y_{\left(t_{j+1}-t_{j}\right)}}
$$

Now we observe that $Z_{t_{j+1}}-Z_{t_{j}}=\Delta Z_{j}=\Delta X_{j}+\Delta Y_{j}$ and that $\Delta X_{j}$ and $\Delta Y_{j}$ are independent. Hence by(9)

$$
\begin{gathered}
F_{\left(Z_{t_{j+1}}-Z_{t_{j}}\right)}=F_{\Delta Z_{j}}=F_{\left(\Delta X_{j}+\Delta Y_{j}\right)}=F_{\Delta X_{j}} * F_{\Delta Y_{j}}=F_{X_{\left(t_{j+1}-t_{j}\right)}} * F_{Y_{\left(t_{j+1}-t_{j}\right)}} \\
=F_{\left(X_{\left(t_{j+1}-t_{j}\right)}+Y_{\left(t_{j+1}-t_{j}\right)}\right)}=F_{Z_{\left(t_{j+1}-t_{j}\right)}}
\end{gathered}
$$

which shows that $Z_{t}$ has stationary increments.

Finally the stochastic continuity follows from the elementary inequality of probability:

$$
P\left(\left|Z_{t}-Z_{S}\right|>a\right) \leq P\left(\left|X_{t}-X_{S}\right|>\frac{a}{2}\right)+P\left(\left|Y_{t}-Y_{S}\right|>\frac{a}{2}\right) \longrightarrow 0 \quad \text { as } \quad t \rightarrow s,
$$

since $X_{t}$ and $Y_{t}$ are Lévy processes. So $Z_{t}$ is a Lévy process. 


\section{Jump processes associated with Lévy processes}

Jump process associated with a cadlag stochastic process has already been defined. Analytic difficulty in manipulating the jump processes arises from the fact that it is possible for them to have unbounded variation:

$$
\sum_{0 £ s £ t}\left|D X_{s}\right|=¥ \text { a.s. }
$$

The way these difficulties are tackled uses the fact that we always have

$$
\sum_{0 £ s £ t}\left|D X_{s}\right|^{2}<¥ \text { a.s. }
$$

when the process,with which the jump process is associated,is a Lévy process .

The first result shows that the jump process, though associated with a Lévy process, is not itself a Lévy process in general. Following characterization of Poisson process through the associated jump process is necessary.

Theorem 4.1 If $N$ is a Lévy process that is increasing a.s. and is such that $\left\{\Delta N_{t}, t \geq 0\right\}$ takes values in $\{0,1\}$, then $N$ is a Poisson process.

A nice proof can be found e.g. in [2].

Proposition 4.1 Let $N=\left\{N_{t}, t \geq 0\right\}$ be a Poisson process. Then for $0 \leq t_{1}<t_{2}<\infty$

$$
P\left(\Delta N_{t_{2}}-\Delta N_{t_{1}}=0 \mid \Delta N_{t_{1}}=1\right) \neq P\left(\Delta N_{t_{2}}-\Delta N_{t_{1}}=0\right)
$$

i.e. $\Delta N=\left\{\Delta N_{t}, t \geq 0\right\}$ can not have independent increments.

Proof: We define the stopping times $T_{0}=0$ and for $n \in N$

$$
T_{n}=\inf \left\{t \geq 0: N_{t}=n\right\} .
$$

It is well known that $T_{n}$, being a sum of exponentials, is Gamma distributed. Now for any $t \geq 0$

$$
\begin{aligned}
P\left(D N_{t}=1\right) & =P\left(N_{t}-N_{t^{-}}=1\right) \\
& =\sum_{n=1}^{¥} P\left(N_{t}-N_{t^{-}}=1, N_{t}=n\right) \\
& =\sum_{n=1}^{¥} P\left(T_{n}=t\right)=0,
\end{aligned}
$$

and

$$
\begin{aligned}
P\left(D N_{t}\right. & =0)=P\left(N_{t}-N_{t^{-}}=0\right) \\
& =\sum_{n=1}^{\ddagger} P\left(N_{t}-N_{t^{-}}=0, N_{t}=n\right)
\end{aligned}
$$




$$
\begin{aligned}
& =\sum_{n=1}^{¥} P\left(T_{n}<t<T_{n+1}\right) \\
& =\sum_{n=1}^{¥} P\left(T_{n+1}>t\right)-P\left(T_{n}{ }^{3} t\right) \\
& =\lim _{n \circledast ¥} P\left(T_{n}>t\right) \\
& =1-\lim _{n \circledast ¥} P\left(T_{n} £ t\right) .
\end{aligned}
$$

That is

$$
\begin{gathered}
P\left(\Delta N_{t}=0\right)=1-\lim _{n \rightarrow \infty} P\left(T_{n}-T_{n-1}+\cdots+T_{2}-T_{1}+T_{1} \leq t\right) \\
\geq 1-\lim _{n \rightarrow \infty} P\left(T_{1} \leq \frac{t}{n}\right)=1 .
\end{gathered}
$$

Now considering these facts, the conditional probability

$$
P\left(\Delta N_{t_{2}}-\Delta N_{t_{1}}=0 \mid \Delta N_{t_{1}}=1\right)=P\left(\Delta N_{t_{2}}=1 \mid \Delta N_{t_{1}}=1\right)=P\left(\Delta N_{t_{2}}=1\right)=0
$$

while the unconditional one is given by

$$
\begin{aligned}
P\left(D N_{t_{2}}-D N_{t_{1}}\right. & =0) \\
& =P\left(D N_{t_{2}}=D N_{t_{1}}=0\right)+P\left(D N_{t_{2}}=D N_{t_{1}}=1\right) \\
& =P\left(D N_{t_{2}}=0\right) P\left(D N_{t_{1}}=0\right)+P\left(D N_{t_{2}}=1\right) P\left(D N_{t_{1}}=1\right) \\
& =P\left(D N_{t_{2}}=0\right) P\left(D N_{t_{1}}=0\right)=1 .
\end{aligned}
$$

Now we will see that we are saved from the difficulty expressed in (13) whenever the process, with which the jump process is associated, is the compound Poisson process.

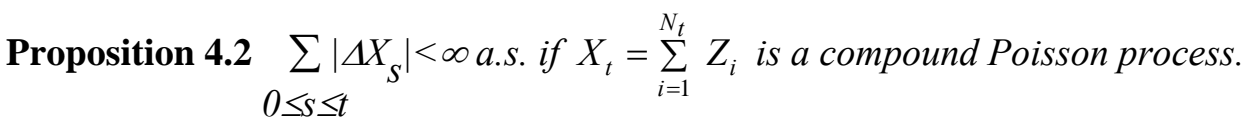

Proof: Since $N_{t}$ is a Poisson process, we have $N_{t}=\sum_{0 £<s<t} D N_{s}$. Now if for some $\omega$, $N_{t}(\omega)=n$ there exists $0<t_{1}(\omega)<\cdots<t_{n}(\omega) \leq t$ such that $\Delta N_{t_{i}}=1$ and $N_{t_{i}}=i, 1 \leq i \leq n$. Then for the compound Poisson process $X_{t}=Z_{1}+\cdots+Z_{N_{t}}$ clearly we have

$$
\sum_{0 \leq s \leq t}\left|\Delta X_{s}\right|=\sum_{i=1}^{n}\left|Z_{i}\right|=\left|Z_{1}\right|+\cdots+\mid Z_{N} N^{\mid<\infty} .
$$


For the general Lévy process we are saved from the same difficulty as seen in the following result.

Proposition 4.3 If $X_{t}$ is a Lévy process then for each $t \geq 0$

$$
\sum_{0 £ s £ t}\left[D X_{s}\right]^{2}<¥ a . s .
$$

The proof of this result yields as a corollary of the celebrated "Lévy-Ito" decomposition. For the proof and much more on this the readers are referred to e.g. [3] and [4].

\section{Conclusion}

The purpose of this paper is to get the idea of interplay between the Lévy processes and the jump processes associated with them. In so doing it has been addressed how properties of Lévy processes help us analyzing and solving difficulties of jump processes associated with the corresponding Lévy process. This paper bridges the gap in the existing literature by addressing the ideas behind constructive proofs in a rigorous way. These ideas are intuitive and can be applied in solving many practical problems.

\section{REFERENCES}

1. Cont, Rama and Tankov, Peter, Financial Modelling With Jump Processes. Chapman \& Hall/CRC Financial Mathematics Seris : London (2004).

2. Applebeum, David, Lévy Processes and Stochastic Calculus. Cambridge University Press: Cambridge, UK (2004).

3. Sato, Ken-Iti, Lévy Processes and Infinitely Divisible Distributions. Cambridge University Press: Cambridge, UK (1999).

4. Bertoin, Jean, Lévy Processes. Cambridge University Press : Cambridge, UK.

5. Winkel, Matthias (2007). Lévy processes and finance. Unpublished Lecture notes. Available at http://www.stats.ox.ac.uk/winkel/ms3b.html Oxford University, UK (1996). 